\title{
Evaluation of changes in shoulder balance and prediction of final shoulder imbalance during growing-rod treatment for early- onset scoliosis
}

Ziyang Liu ${ }^{1 \dagger}$, Tie Liu ${ }^{1 \dagger}$, Yong Hai ${ }^{1 *}$, Lingyun Wu² , Junrui Jonathan $\mathrm{Hai}^{3}$, Kang Gao ${ }^{4}$, Xuanrong Guo ${ }^{5}$, Honghao Yang ${ }^{1}$, Nan Kang ${ }^{1}$ and Fan Zhao ${ }^{1}$

\begin{abstract}
Background: Obtaining and maintaining final shoulder balance after the entire treatment course is essential for early-onset scoliosis (EOS) patients. The relatively small number of growing-rod (GR) graduates who complete final fusion has resulted in an overall paucity of research on the GR treatment of EOS and a lack of research on the shoulder balance of EOS patients during GR treatment.

Methods: Twenty-four consecutive patients who underwent GR treatment until final fusion were included. Radiographic shoulder balance parameters, including the radiographic shoulder height (RSH), clavicle angle (CA), and $\mathrm{T1}$ tilt angle (T1T), before and after each step of the entire treatment were measured. Shoulder balance changes from GR implantation to the last follow-up after final fusion were depicted and analysed. Demographic data, surgical-related factors, and radiographic parameters were analysed to identify risk factors for final shoulder imbalance. The shoulder balance of patients at different time points was further analysed to explore the potential effect of the series of GR treatment steps on shoulder balance.

Results: The RSH showed substantial improvement after GR implantation $(P=0.036)$, during the follow-up period after final fusion $(P=0.021)$ and throughout the entire treatment $(P=0.011)$. The trend of change in the CA was similar to that of the RSH, and the T1T improved immediately after GR implantation ( $P=0.037)$. Further analysis indicated that patients with shoulder imbalance before final fusion showed significantly improved shoulder balance after fusion $(P=0.045)$, and their RSH values at early postfusion and the final follow-up did not show statistically significant differences from those in the prefusion shoulder balance group $(P>0.05)$. Early postfusion shoulder imbalance (odds ratio (OR): 19.500; 95\% confidence interval $(\mathrm{Cl})=1.777-213.949 ; P=0.015$ ) was identified as an independent risk factor for final shoulder imbalance.

(Continued on next page)
\end{abstract}

\footnotetext{
* Correspondence: prof.haiyong@yahoo.com; spinesurgeon@163.com

†Ziyang Liu and Tie Liu contributed equally to this work.

'Department of Orthopedic Surgery, Beijing Chaoyang Hospital, Capital

Medical University, Gongti North Rd, No. 8, Beijing 100020, China

Full list of author information is available at the end of the article
}

(c) The Author(s). 2021 Open Access This article is licensed under a Creative Commons Attribution 4.0 International License, which permits use, sharing, adaptation, distribution and reproduction in any medium or format, as long as you give appropriate credit to the original author(s) and the source, provide a link to the Creative Commons licence, and indicate if changes were made. The images or other third party material in this article are included in the article's Creative Commons licence, unless indicated otherwise in a credit line to the material. If material is not included in the article's Creative Commons licence and your intended use is not permitted by statutory regulation or exceeds the permitted use, you will need to obtain permission directly from the copyright holder. To view a copy of this licence, visit http://creativecommons.org/licenses/by/4.0/ The Creative Commons Public Domain Dedication waiver (http://creativecommons.org/publicdomain/zero/1.0/) applies to the data made available in this article, unless otherwise stated in a credit line to the data. 
(Continued from previous page)

Conclusions: Shoulder balance could be improved by GR implantation but often changes during the multistep lengthening process, and the final result is relatively unpredictable. Final fusion could further adjust the prefusion shoulder imbalance. Focusing on the prefusion shoulder balance of GR graduates and providing patients with early shoulder balance after fusion might be necessary.

Keywords: Shoulder balance, Early-onset scoliosis, Growing rod, Spinal fusion, Postoperative shoulder imbalance

\section{Background}

Good surgical outcomes in patients with scoliosis include the sufficient correction of curves in three planes and prevention of further progression, as well as good shoulder balance and trunk balance [1-3]. Shoulder balance influences not only patient appearance and satisfaction but also potential complications, such as adding-on, trunk shifting, and reoperation [2-7]. Thus, preventing postoperative shoulder imbalance requires particular attention. Previous studies have reported various risk factors for postoperative shoulder imbalance but are mostly based on adolescent idiopathic scoliosis [2, 4, 5, 8-11]. The risk factors for postoperative shoulder imbalance in early-onset scoliosis (EOS) are still unknown.

Growing-rod (GR) surgery is indicated for EOS patients when curves are of sufficient magnitude and when conservative treatment methods have failed [12-14]. The entire GR treatment has been proven to obtain optimal correction results and excellent growth potential [12-16], but the effect on shoulder balance has remained understudied. Uzümcügil et al. [17] and Atici et al. [18, 19] evaluated the effect of GR on shoulder balance during treatment periods. However, only a few patients in previous studies were GR graduates who underwent final fusion. Final fusion is considered to be the first choice for the last step of GR treatment for most EOS patients, and although it remains controversial, most patients who have completed final fusion have achieved definitive curative effects [20-22]. Focusing on GR graduates who have completed final fusion is of great significance for investigating the effect of GR on final shoulder balance. In this study, all patients included were GR graduates who had completed final fusion, a distinction lacking in previous studies.

The purpose of this study was to explore the effect of GR on shoulder balance in GR graduates and to identify possible predictors for final shoulder balance.

\section{Methods}

\section{Inclusion criteria}

Twenty-four consecutive EOS patients who underwent the entire series of surgical treatments, including GR implantation, multiple lengthening surgeries, and final fusion, at our institution from March 2008 to July 2018 were studied. Patients who underwent other spinal surgeries were excluded.

\section{Parameter measurements}

We obtained radiographic parameters by measuring the standing full-length spine films taken at predetermined time points: before and early after GR implantation, before and after multiple lengthening surgeries, before and early after final fusion, and at the last follow-up. Two observers performed measurements independently using the Picture Archiving and Communication System (PACS) technology (GE Medical Systems, 2006).

The radiographic shoulder height ( $\mathrm{RSH}$ ) was obtained by measuring the height difference of the soft tissue shadow above the acromioclavicular joint, and absolute $\mathrm{RSH}$ values were used to represent the severity of shoulder imbalance [23, 24]. Shoulder balance conditions were categorised based on the RSH: balance $(<2 \mathrm{~cm})$, imbalance (mild imbalance $(2-3 \mathrm{~cm})$, and severe imbalance $(>3 \mathrm{~cm}))[2,4,5,9,10]$. In addition, the T1 tilt angle (T1T) was defined as the angle between the upper endplate of the T1 vertebral body and the horizontal line, while the clavicle angle (CA) was defined as the angle between the tangent line touching the highest point of each clavicle and the horizontal line [3-6, 8-10, 23].

The major curve $(\mathrm{MC})$, the proximal thoracic curve (PTC), the cervical 7 plumb line-centre sacral vertical line (C7PL-CSVL), the cervical 7 plumb line-sagittal vertebral axis (C7PL-SVA), thoracic kyphosis (TK), and lumbar lordosis (LL) were also measured.

\section{Satisfaction questionnaire}

The questionnaire (see supplementary information) was modified from the Spinal Appearance Questionnaire (SAQ) [25] and that of Kuklo et al. [23] according to the characteristics of GR treatment to assess parental perception and satisfaction of patients' shoulder balance. It was distributed to fifteen parents of the included patients who had finished the treatment in the last 6 years.

\section{Statistical analysis}

Intraclass correlation coefficients (ICCs) were calculated to assess the interobserver and intraobserver reliability of the measurements performed by the observers. Categorical variables were compared by Fisher's exact test. 
Comparisons between two different groups were performed by the Mann-Whitney $U$ test or independent $t$ test; comparisons between different time points were performed by repeated-measures ANOVA. Multiple time point analyses were performed to describe the changes in shoulder balance during GR treatment.

To investigate the relationships between patient characteristics and clinical outcomes, variables that exhibited significant differences in univariate comparisons (the threshold for variables included in the multivariate regression analysis was $P=0.10$ ) were included in the stepwise logistic regression to identify the risk factors for shoulder imbalance at follow-up.

Analyses were performed by SPSS 25.0 (SPSS, Chicago, IL). A $P$ value of less than 0.05 was considered statistically significant.

\section{Results}

\section{Demographics}

Demographic data are summarised in Table 1. Due to failure to control spinal deformity, severe coronal decompensation and rod breakage, single rod in six patients were replaced with dual rods during the lengthening process. Eleven patients had dual rods before final fusion, and single-rod configurations remained in thirteen patients.

\section{Radiographic measurements}

Radiographic measurements are summarised in Table 2. The analysis revealed significant improvement in the MC throughout the entire treatment $(P<0.001)$. In addition, the average PTC and TK significantly decreased (PTC: $P<0.001$; TK: $P=0.041$ ).

\section{Changes in shoulder balance}

The intraobserver ICC was 0.88 , and the interobserver ICC was 0.83 [26].

RSH values at different time points are plotted in Fig. 1a. The average RSH at preimplantation, early postimplantation, prefusion, early postfusion, and final follow-up was $23.8 \pm 16.0 \mathrm{~mm}, 17.1 \pm 8.6 \mathrm{~mm}, 18.6 \pm$ $11.0 \mathrm{~mm}, 16.4 \pm 9.5 \mathrm{~mm}$, and $13.2 \pm 10.7 \mathrm{~mm}$, respectively. The analysis revealed statistically significant improvements in the RSH after GR implantation $(P=$ $0.036)$, during the follow-up period after final fusion $(P=0.021)$, and throughout the entire treatment $(P=$ 0.011). The RSH during the lengthening procedures, however, did not change significantly $(P>0.05)$, and there was no significant difference in the RSH between before fusion and before implantation $(P>0.05)$. The changes in the CA and T1T are shown in Fig. 1b, c. As shown in Fig. 1b, the average CA decreased significantly after GR implantation $(P=0.008)$, during the follow-up period after fusion $(P=0.024)$, and after the entire
Table 1 Demographics

\begin{tabular}{|c|c|}
\hline Descriptive items & Mean \pm SD or Number (\%) \\
\hline \multicolumn{2}{|l|}{ Sex } \\
\hline Male & $6(25.0 \%)$ \\
\hline Female & $18(75.0 \%)$ \\
\hline \multicolumn{2}{|l|}{ Diagnosis } \\
\hline Idiopathic & $10(41.7 \%)$ \\
\hline Congenital & $9(37.5 \%)$ \\
\hline Other & $5(20.8 \%)$ \\
\hline Age (implantation) (year) & $9.8 \pm 2.3$ \\
\hline Age (fusion) (year) & $13.0 \pm 1.5$ \\
\hline \multicolumn{2}{|l|}{ Risser sign (implantation) (0/1) } \\
\hline 0 & $21(87.5 \%)$ \\
\hline 1 & $3(12.5 \%)$ \\
\hline \multicolumn{2}{|l|}{ Risser sign (fusion) (4/5) } \\
\hline 4 & $4(16.7 \%)$ \\
\hline 5 & $20(83.3 \%)$ \\
\hline Treatment duration (year) & $3.2 \pm 1.4$ \\
\hline No. of lengthening surgeries & $2.5 \pm 1.5$ \\
\hline \multicolumn{2}{|l|}{ Implantation rod(s) } \\
\hline Single & 19 (79.2\%) \\
\hline Dual & $5(20.8 \%)$ \\
\hline \multicolumn{2}{|l|}{ UIV (Growing rods) } \\
\hline T2 and above & $16(66.7 \%)$ \\
\hline T3 and below & $8(33.3 \%)$ \\
\hline \multicolumn{2}{|l|}{ UIV (Fusion) } \\
\hline T2 and above & $14(58.3 \%)$ \\
\hline T3 and below & $10(41.7 \%)$ \\
\hline \multicolumn{2}{|l|}{ LIV (Growing rods) } \\
\hline L3 and above & $10(41.7 \%)$ \\
\hline L4 and below & $14(58.3 \%)$ \\
\hline \multicolumn{2}{|l|}{ LIV (Fusion) } \\
\hline L3 and above & $17(70.8 \%)$ \\
\hline L4 and below & $7(29.2 \%)$ \\
\hline \multicolumn{2}{|c|}{ Upper instrument (Growing rods) } \\
\hline Hook/hybrid & $16(66.7 \%)$ \\
\hline No hook & $8(33.3 \%)$ \\
\hline \multicolumn{2}{|l|}{ Upper instrument (Fusion) } \\
\hline Hook/hybrid & $5(20.8 \%)$ \\
\hline No hook & 19 (79.2\%) \\
\hline
\end{tabular}

UIV upper instrumented vertebrae; $L I V$ lower instrumented vertebrae

treatment $(P=0.003)$. The T1T showed a significant decrease after GR implantation ( $P=0.037$; Fig. 1c).

Figure 2 shows a plot of the number of patients with balanced shoulders at different time points. At the five time points mentioned above, the number of patients 
Table 2 Radiographic parameters from preimplantation to final follow-up for the entire study population

\begin{tabular}{lllllll}
\hline & MC $\left(^{\circ}\right)$ & PTC $\left(^{\circ}\right)$ & TK $\left(^{\circ}\right)$ & LL $\left(^{\circ}\right)$ & C7PL-CSVL (cm) & C7PL-SVA (cm) \\
\hline Preimplantation & $96 \pm 22$ & $43 \pm 18$ & $47 \pm 24$ & $48 \pm 14$ & $3.0 \pm 2.6$ & $3.0 \pm 2.0$ \\
Postimplantation & $51 \pm 20$ & $30 \pm 13$ & $33 \pm 15$ & $41 \pm 14$ & $2.4 \pm 1.8$ & $3.2 \pm 1.8$ \\
Prefusion & $59 \pm 20$ & $30 \pm 12$ & $41 \pm 15$ & $57 \pm 14$ & $2.4 \pm 1.7$ & $2.7 \pm 2.1$ \\
Follow-up & $38 \pm 17$ & $21 \pm 8$ & $35 \pm 12$ & $52 \pm 12$ & $2.7 \pm 1.6$ & $3.2 \pm 2.3$ \\
\hline
\end{tabular}

MC major curve; PTC proximal thoracic curve; TK thoracic kyphosis; LL lumbar lordosis; C7PL cervical 7 plumb line; CSVL centre sacral vertical line; SVA sagittal vertebral axis

with balanced shoulders was 10 (42\%), 14 (58\%), 11 (46\%), 14 (58\%), and 17 (71\%).

To further identify the time points with potentially predictable final shoulder balance and further analyse the potential impact of each step of GR treatment on shoulder balance, the patients were divided into two (shoulder balance and shoulder imbalance) groups at the aforementioned five time points. Figure 3 shows a significant difference in the final RSH between the early postfusion shoulder balance and imbalance groups $(P=$ 0.008). Further analysis results of the postimplantation groups are shown in Fig. 4a. Although the RSH in the postimplantation shoulder imbalance group did not show a significant improvement from postimplantation to prefusion $(P>0.05)$, the RSH in the two groups no longer showed a significant difference between prefusion and the final follow-up $(P>0.05)$. The results of further analysis of the prefusion groups are shown in Fig. 4b. For patients with shoulder imbalance before fusion, their RSH was improved immediately after fusion $(P=0.045)$, and there was no longer a significant difference from the prefusion shoulder balance group $(P>$ 0.05).

\section{Variable analysis}

Seven (29\%) patients developed radiographic shoulder imbalance at the last follow-up based on the diagnostic criteria. The results of the univariate analysis showed that potential risk factors for shoulder imbalance included late acceptance of GR implantation $(P=0.094)$ and final fusion $(P=0.091)$, upper instrumented vertebrate (UIV) below T2 at final fusion $(P=0.085)$, prefusion sagittal imbalance $(P=0.074)$, a large early postfusion $\mathrm{RSH}(P=0.002)$, a large early postfusion CA $(P=0.082)$, and a rating of radiographic shoulder imbalance $(P=0.009)$ (Table 3$)$. In the multivariate logistic stepwise regression analysis, early postfusion shoulder imbalance (odds ratio (OR): 19.500; 95\% confidence interval $(\mathrm{CI})=1.777-213.949$; $P=0.015)$ was found to be an independent risk factor for final shoulder imbalance.

\section{Questionnaire}

Thirteen $(87 \%, 13 / 15)$ valid questionnaires were received. Four (31\%) patients had radiographic shoulder imbalance at the last follow-up and were considered 'mildly unbalanced'. Nine (69\%) patients were corrected

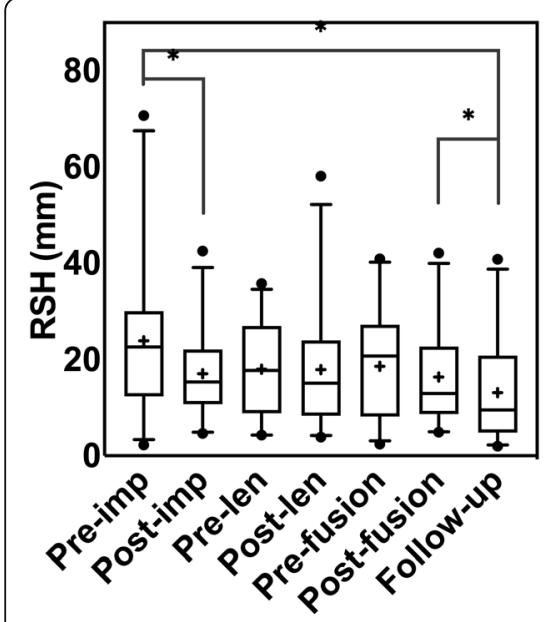

A

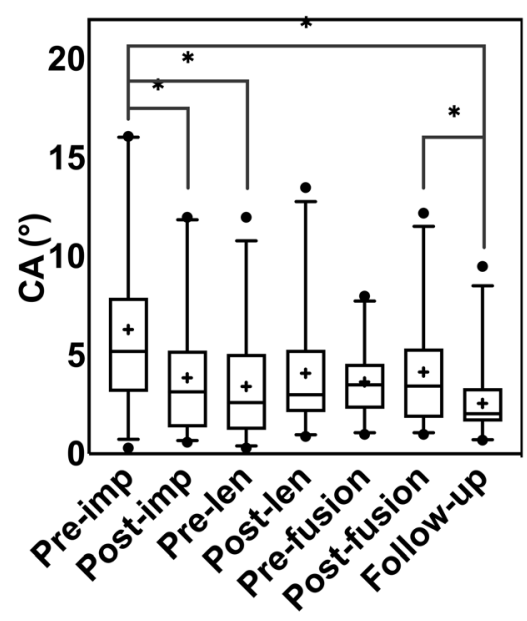

B

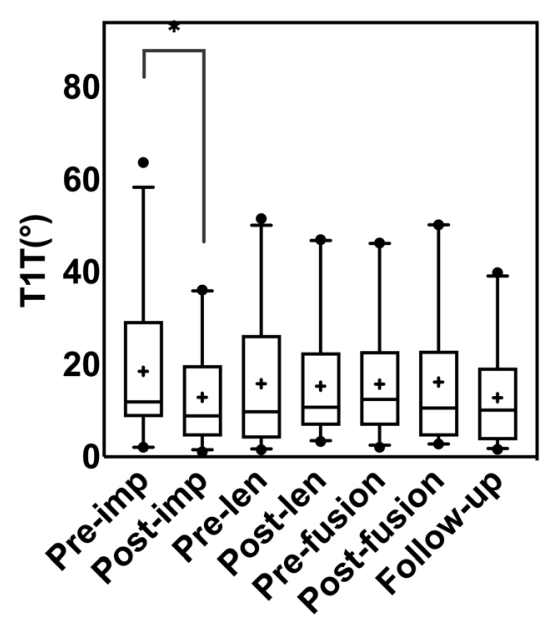

C

Fig. 1 Changes in the radiographic shoulder balance of EOS patients throughout the entire growing rod treatment period. a RSH (b) CA (c) T1T.*Statistically significant. +: mean value. $\bullet$ : extreme values 


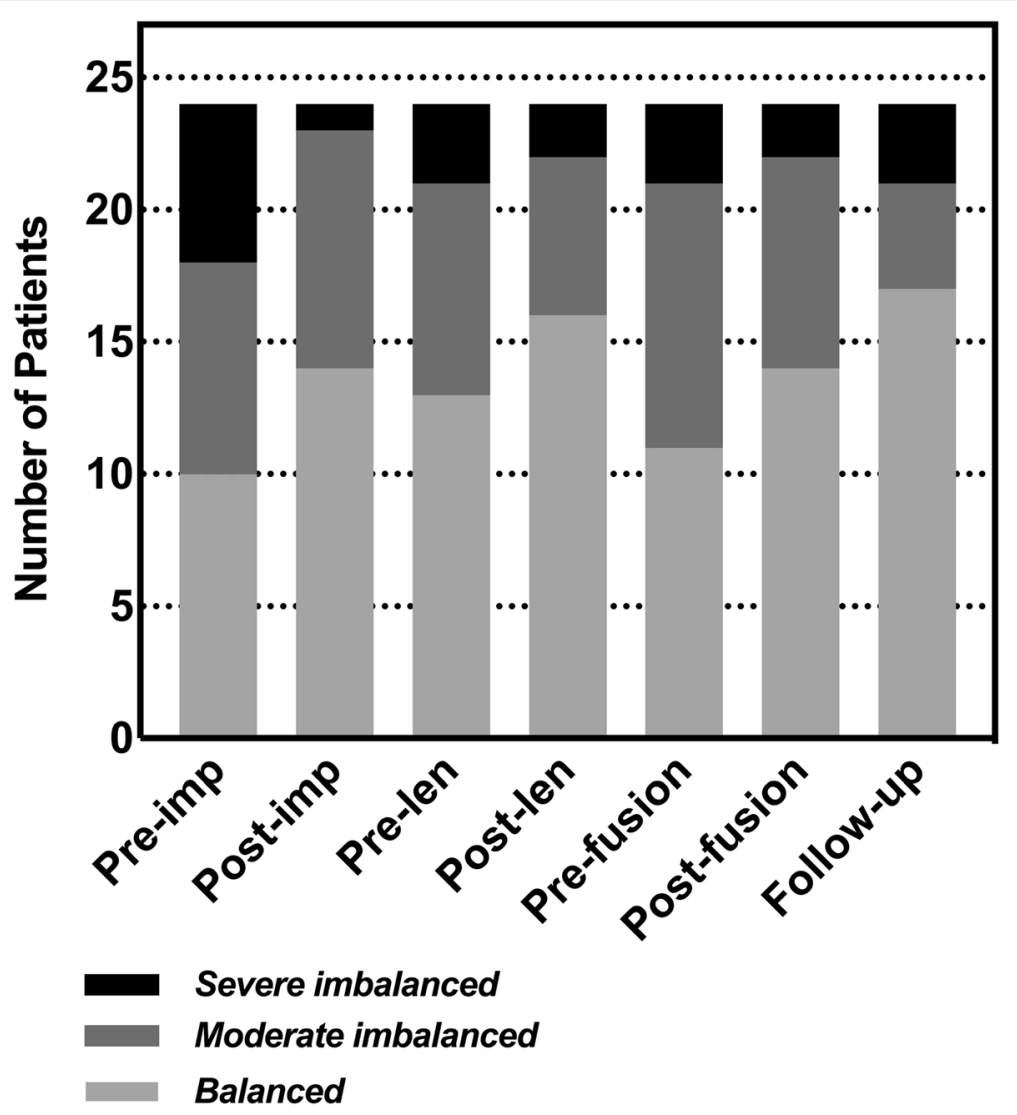

Fig. 2 Number of patients with different shoulder balance statuses during the growing rod treatment period

to radiographic shoulder balance (6 (67\%) were considered 'balanced', and three (33\%) were considered 'mildly unbalanced'). There was no statistically significant difference in the parental evaluation of shoulder balance between patients with radiographic shoulder balance and imbalance $(P=0.228)$. Interestingly, all parents $(100 \%)$ agreed that GR treatment improved the shoulder balance of the patients. All parents (100\%) were satisfied with the final shoulder condition of patients $(9$ (69\%) chose 'basically satisfied', 4 (31\%) chose 'very satisfied'): Parental satisfaction did not show statistically significant differences between patients with final radiographic shoulder imbalance and final radiographic shoulder balance $(P=0.109)$.

\section{Discussion}

Immediately after GR implantation, the mean RSH showed a significant decrease $(P=0.036$; Fig. 1a), and the number of patients with shoulder balance increased from 10 to 14. Furthermore, the CA $(P=0.008)$ and T1T $(P=0.037)$ improved immediately after $\mathrm{GR}$ implantation. These results suggested that GR implantation improved shoulder balance. Studies by Uzümcügil et al. [17] and Atici et al. $[18,19]$ reported that GR had a positive effect on shoulder balance. Our findings are consistent with these previous reports. Adjustment of the shoulder balance is part of adjacent compensation, which is often triggered by deformity correction [27, 28]. In view of that, we attributed the adjustment of shoulder balance to the significant correction of the PTC and MC (Table 2) $[2,10,29]$.

After multiple lengthening procedures, there was no longer a statistically significant difference in the RSH between the patients with preimplantation shoulder balance and those with shoulder imbalance (Fig. 4a), and there was no statistically significant difference in the radiographic shoulder balance parameters of all patients between before fusion and before implantation $(P>0.05$; Fig. 1abc). In general, regular lengthening not only prevents the deterioration of spinal deformities and treats complications but also unleashes spine growth potential and readjusts the balance $[20,30,31]$. However, according to the aforementioned studies and the current study, regular lengthening had no significant effect on improving shoulder balance.

Final fusion was recommended for most GR graduates, but in previous studies, only a small number of patients were GR graduates who underwent final fusion [17-19]. 


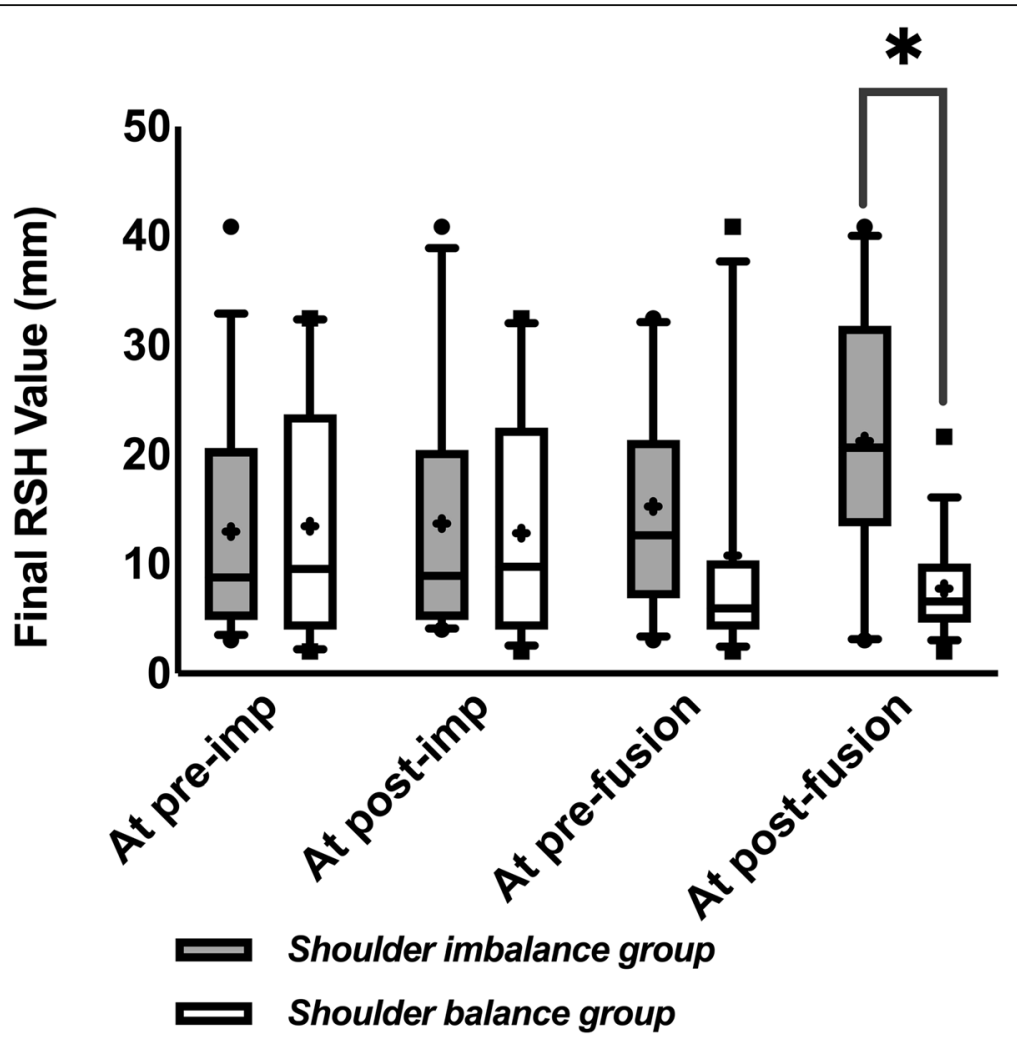

Fig. 3 Comparison of the final RSH between patients grouped by shoulder balance status at preimplantation, postimplantation, prefusion, and early postfusion. Grey bar: shoulder imbalance group. White bar: shoulder balance group. *Statistically significant. +: mean value. $\bullet$ m: extreme values

In this study, all patients included were GR graduates who completed final fusion and had relatively complete follow-up data. Further analysis showed that final fusion significantly improved shoulder balance in patients with prefusion shoulder imbalance (Fig. 4b). In addition, the number of patients with severe shoulder imbalance decreased after final fusion (Fig. 2). These results might suggest that final fusion enabled rebalancing in patients with shoulder imbalance. In general, final fusion can help patients not only stop the high complication rate during multiple lengthening procedures and further correct deformities but also adjust trunk imbalance and achieve better biomechanical fixation [20, 22, 30, 31]. Furthermore, it is encouraging that final fusion achieved good results for improving prefusion shoulder imbalance according to the results.

Our study revealed a significant difference in the RSH at the last follow-up compared with immediately after fusion $(P=0.021$; Fig. 1a), and the number of patients with balanced shoulders increased during the follow-up period after fusion (Fig. 2). A similar trend was found in the CA $(P=0.024$; Fig. $1 \mathrm{~b})$. These results might suggest that GR graduates might experience 'spontaneous correction of shoulder balance' after final fusion. Although there is no uniform language that describes this conjecture in the literature, this phenomenon has been reported in patients with adolescent idiopathic scoliosis undergoing spinal fusion $[4,10,23,27]$. However, although it has not been previously reported in EOS patients, it might exist in EOS patients who complete final fusion given the similarities between segmental fusion for other types of scoliosis surgery and final fusion. The selective instrumented segments in final fusion often preserve motion segments for the postoperative spine, which enables postoperative balance restoration $[6,32-$ 35]. In addition, according to previous reports, the early postoperative shoulder balance status might be affected by postoperative pain and malposture, which might improve once postoperative pain is relieved [35].

Perfect postoperative neutral shoulder balance is not always achieved, but this does not mean that shoulder balance is not favourably improved by surgery. A certain degree of neutral shoulder bias can exist even in the normal population. Previous studies on shoulder balance parameters in normal people without scoliosis have shown that most people's shoulder balance is not in the absolute neutral position, but the vast majority of people are at approximately the absolute neutral level $[28,36-$ 38]. Currently, the maximum sample size that has been used in a study of a normal population (232 adolescents) 


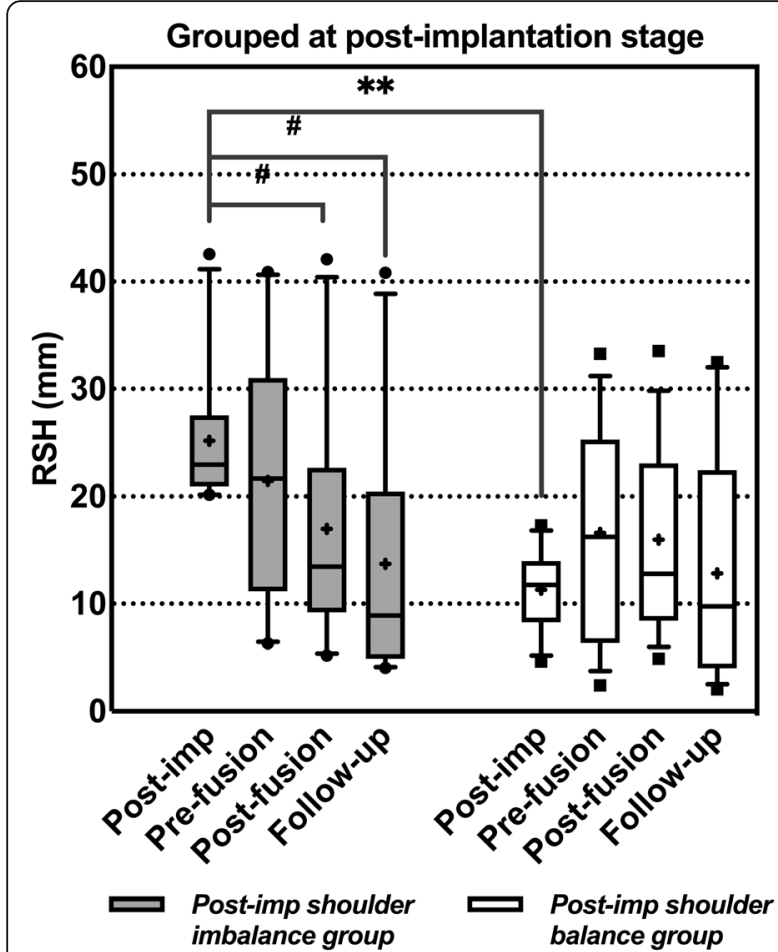

A

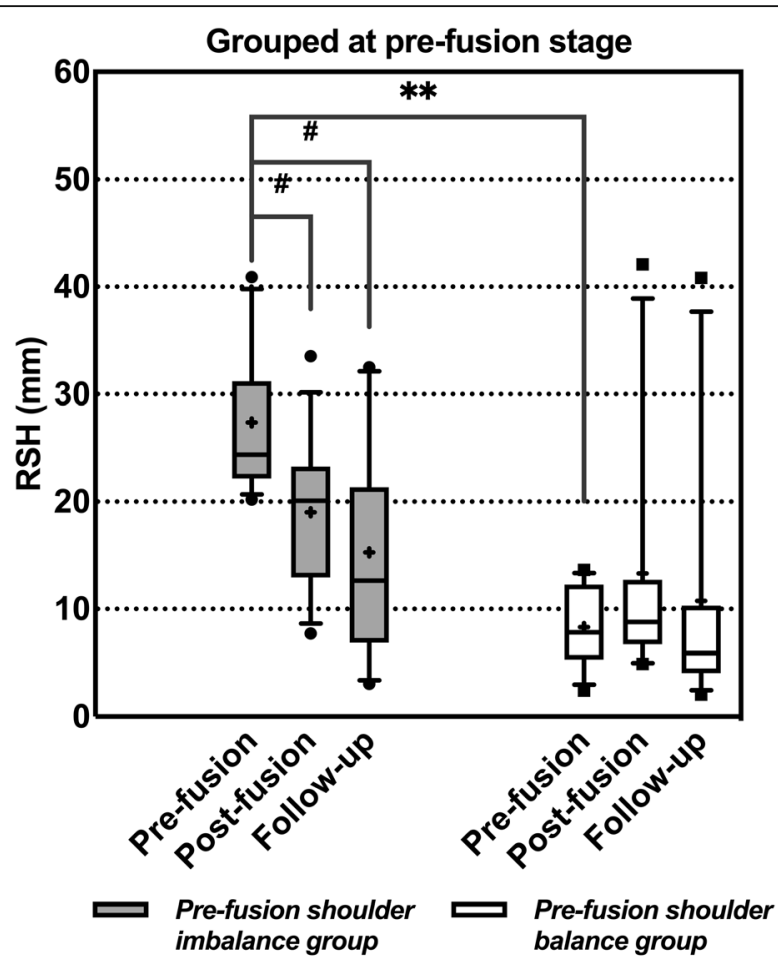

B

Fig. 4 Changes in the RSH of patients in the shoulder balance group and shoulder imbalance group. Patients grouped at (a) postimplantation and (b) prefusion. Grey bar: shoulder imbalance group. White bar: shoulder balance group. *Statistically significant differences between groups $\left.{ }^{* *} P<0.001\right)$. \#Statistically significant improvement in the group. +: mean value. •n: extreme values

has shown that the average RSH in the normal population is $0.9 \mathrm{~cm}$ [28]. In our study, the patients' mean RSH improved from $23.8 \pm 16.0 \mathrm{~mm}$ at preimplantation to $13.2 \pm 10.7 \mathrm{~mm}$ at the final follow-up, showing a statistically significant improvement and tending toward the neutral shoulder balance level in the general population.

In this study, shoulder imbalance early after fusion was identified as a definite risk factor for final shoulder imbalance in GR graduates. Early postoperative RSH imbalance was previously reported to be a primary predictor of final shoulder imbalance [2]. Notably, early postoperative shoulder imbalance as an independent risk factor seems to contradict the spontaneous correction of shoulder balance after final fusion. However, this study demonstrated limited spontaneous recovery of shoulder balance after final fusion and that not every patient with early postoperative shoulder imbalance eventually showed an improvement, which is consistent with previous literature $[4,10,27]$. Other potential risk factors, including the UIV of final fusion, age at implantation, age at final fusion, and prefusion C7PL-SVA, were identified as not statistically significant. Whether the above factors affect the final shoulder balance of patients with EOS still needs to be further explored.
This study has several limitations. First, the aetiological diversity of EOS made it difficult to assess the efficacy considering the substantial variation in treatment indications and methods among different individuals. The patients included in this study were not only idiopathic but had a variety of curve types. Furthermore, there were some confounding factors leading to bias in this study, including different types of growing rods (single/dual rod(s)) and the replacement of a single rod by dual rods during the GR lengthening process. Moreover, the average follow-up period was relatively short, and more patients in this study were treated with a single rod rather than the most commonly used dual rod, which limited the significance of this study. A prospective multicentre study that focuses on a particular aetiology or type of curvature with more extended follow-up periods and a larger sample size should be developed to overcome the above limitations. Additionally, with the emergence of cosmetic shoulder balance research, it is worthwhile to study patient selfperception, self-evaluations of treatment efficacy, and satisfaction in addition to parameter accuracy and surgical outcomes.

Despite its shortcomings, this study still contributed several novel insights. This study was the first to observe 
Table 3 Univariate analysis of shoulder imbalance risk factors

\begin{tabular}{|c|c|c|c|}
\hline Parameters & Balanced shoulder & Imbalanced shoulder & $P$ \\
\hline \multicolumn{4}{|l|}{ Demographics } \\
\hline Sex (male/female) & $4 / 13$ & $2 / 5$ & 1.000 \\
\hline Diagnosis (congenital/not) & $5 / 12$ & $4 / 3$ & 0.356 \\
\hline Age at implantation & $9.3 \pm 2.3$ & $11.0 \pm 1.6$ & 0.094 \\
\hline Age at fusion & $12.7 \pm 1.5$ & $13.9 \pm 1.2$ & 0.091 \\
\hline Risser sign at implantation (0/1) & $15 / 2$ & $6 / 1$ & 1.000 \\
\hline Risser sign at fusion (4/5) & $3 / 14$ & $1 / 6$ & 1.000 \\
\hline Treatment duration (y) & $3.4 \pm 1.6$ & $2.8 \pm 1.0$ & 0.429 \\
\hline Left/right curve & $4 / 13$ & $3 / 4$ & 0.374 \\
\hline \multicolumn{4}{|l|}{ Surgery-related } \\
\hline No. of lengthening surgeries & $2.5 \pm 1.7$ & $2.4 \pm 1.0$ & 0.885 \\
\hline \multicolumn{4}{|l|}{ Single/dual rod(s) } \\
\hline Implantation & $13 / 4$ & $6 / 1$ & 1.000 \\
\hline Prefusion & $8 / 9$ & $5 / 2$ & 0.386 \\
\hline \multicolumn{4}{|l|}{ UIV(T2 and above/T3 and below) } \\
\hline Growing rods & $12 / 5$ & $4 / 3$ & 0.647 \\
\hline Fusion & $12 / 5$ & $2 / 5$ & 0.085 \\
\hline \multicolumn{4}{|l|}{ LIV(L3 and above/L4 and below) } \\
\hline Growing rods & $8 / 9$ & $2 / 5$ & 0.653 \\
\hline Fusion & $12 / 5$ & $5 / 2$ & 1.000 \\
\hline \multicolumn{4}{|l|}{ Upper instrument (hook or hybrid/no hook) } \\
\hline Growing rods & $11 / 6$ & $5 / 2$ & 1.000 \\
\hline Fusion & $3 / 14$ & $2 / 5$ & 0.608 \\
\hline Fixation of the apical region/not at fusion & $11 / 6$ & $3 / 4$ & 0.393 \\
\hline Osteotomy/not at fusion & $3 / 14$ & $2 / 5$ & 0.608 \\
\hline \multicolumn{4}{|l|}{ Radiographic } \\
\hline \multicolumn{4}{|l|}{ MC Cobb angle $\left(^{\circ}\right)$} \\
\hline Preimplantation & $98 \pm 20$ & $93 \pm 26$ & 0.637 \\
\hline Postimplantation & $53 \pm 18$ & $45 \pm 26$ & 0.359 \\
\hline Prefusion & $60 \pm 19$ & $57 \pm 24$ & 0.756 \\
\hline Follow-up & $41 \pm 17$ & $32 \pm 18$ & 0.252 \\
\hline Change & $57 \pm 12$ & $61 \pm 17$ & 0.506 \\
\hline \multicolumn{4}{|l|}{ PTC Cobb angle $\left(^{\circ}\right)$} \\
\hline Preimplantation & $44 \pm 20$ & $42 \pm 15$ & 0.815 \\
\hline Postimplantation & $30 \pm 14$ & $29 \pm 10$ & 0.947 \\
\hline Prefusion & $30 \pm 14$ & $30 \pm 9$ & 0.890 \\
\hline Follow-up & $21 \pm 8$ & $21 \pm 6$ & 0.895 \\
\hline Change & $22 \pm 14$ & $21 \pm 13$ & 0.887 \\
\hline \multicolumn{4}{|l|}{ Flexibility (preimplantation) } \\
\hline PTC & $38 \pm 27$ & $29 \pm 12$ & 0.433 \\
\hline MC & $19 \pm 17$ & $19 \pm 15$ & 0.976 \\
\hline \multicolumn{4}{|l|}{ Thoracic kyphosis } \\
\hline Preimplantation & $46 \pm 25$ & $52 \pm 25$ & 0.575 \\
\hline Postimplantation & $34 \pm 15$ & $31 \pm 17$ & 0.689 \\
\hline
\end{tabular}


Table 3 Univariate analysis of shoulder imbalance risk factors (Continued)

\begin{tabular}{|c|c|c|c|}
\hline Parameters & Balanced shoulder & Imbalanced shoulder & $P$ \\
\hline Prefusion & $39 \pm 15$ & $47 \pm 12$ & 0.239 \\
\hline Follow-up & $33 \pm 12$ & $39 \pm 12$ & 0.272 \\
\hline Change & $13 \pm 22$ & $13 \pm 19$ & 0.983 \\
\hline \multicolumn{4}{|l|}{ Lumbar lordosis } \\
\hline Preimplantation & $48 \pm 15$ & $48 \pm 14$ & 0.980 \\
\hline Postimplantation & $40 \pm 13$ & $44 \pm 16$ & 0.511 \\
\hline Prefusion & $56 \pm 14$ & $61 \pm 13$ & 0.384 \\
\hline Follow-up & $53 \pm 10$ & $52 \pm 16$ & 0.888 \\
\hline Change & $-5 \pm 18$ & $-4 \pm 17$ & 0.955 \\
\hline \multicolumn{4}{|l|}{ C7PL-CSVL (cm) } \\
\hline Preimplantation & $3.1 \pm 2.4$ & $3.0 \pm 3.2$ & 0.936 \\
\hline Postimplantation & $2.4 \pm 1.6$ & $2.5 \pm 2.4$ & 0.896 \\
\hline Prefusion & $2.5 \pm 1.6$ & $2.1 \pm 1.8$ & 0.559 \\
\hline Follow-up & $2.9 \pm 1.6$ & $2.2 \pm 1.7$ & 0.392 \\
\hline Change & $0.2 \pm 3.4$ & $0.8 \pm 3.0$ & 0.699 \\
\hline \multicolumn{4}{|l|}{ C7PL-SVA (cm) } \\
\hline Preimplantation & $2.7 \pm 2.0$ & $3.7 \pm 2.0$ & 0.303 \\
\hline Postimplantation & $3.3 \pm 1.9$ & $2.9 \pm 1.4$ & 0.641 \\
\hline Prefusion & $2.2 \pm 1.8$ & $3.9 \pm 2.5$ & 0.074 \\
\hline Follow-up & $3.0 \pm 2.4$ & $3.5 \pm 2.0$ & 0.685 \\
\hline Change & $-0.3 \pm 3.2$ & $0.2 \pm 2.6$ & 0.722 \\
\hline \multicolumn{4}{|l|}{ Shoulder Balance } \\
\hline \multicolumn{4}{|l|}{$\mathrm{RSH}(\mathrm{mm})$} \\
\hline Preimplantation & $23.7 \pm 16.1$ & $24.2 \pm 16.9$ & 0.942 \\
\hline Postimplantation & $16.7 \pm 5.9$ & $18.1 \pm 13.8$ & 0.807 \\
\hline Prelengthening & $18.6 \pm 10.2$ & $16.7 \pm 9.1$ & 0.680 \\
\hline Postlengthening & $18.4 \pm 13.6$ & $16.9 \pm 6.7$ & 0.792 \\
\hline Prefusion & $18.3 \pm 11.8$ & $19.5 \pm 9.8$ & 0.806 \\
\hline Postfusion & $12.5 \pm 6.4$ & $25.8 \pm 9.5$ & 0.002 \\
\hline Follow-up & $7.3 \pm 4.0$ & $27.5 \pm 7.7$ & $<0.001$ \\
\hline \multicolumn{4}{|c|}{$\begin{array}{l}\text { RSB classification } \\
\text { (balanced/unbalanced) }\end{array}$} \\
\hline Preimplantation & $7 / 10$ & $4 / 3$ & 0.659 \\
\hline Postimplantation & $10 / 7$ & $4 / 3$ & 1.000 \\
\hline Prelengthening & $9 / 8$ & $5 / 2$ & 0.653 \\
\hline Postlengthening & $11 / 6$ & $5 / 2$ & 1.000 \\
\hline Prefusion & $9 / 8$ & $2 / 5$ & 0.386 \\
\hline Postfusion & $13 / 4$ & $1 / 6$ & 0.009 \\
\hline Follow-up & $17 / 0$ & $0 / 7$ & $<0.001$ \\
\hline \multicolumn{4}{|l|}{ Other Parameters } \\
\hline \multicolumn{4}{|l|}{$\mathrm{CA}\left({ }^{\circ}\right)$} \\
\hline Preimplantation & $6.8 \pm 4.2$ & $5.1 \pm 4.2$ & 0.392 \\
\hline Postimplantation & $4.0 \pm 2.9$ & $3.6 \pm 4.2$ & 0.814 \\
\hline Prefusion & $3.8 \pm 2.0$ & $3.3 \pm 1.3$ & 0.926 \\
\hline
\end{tabular}


Table 3 Univariate analysis of shoulder imbalance risk factors (Continued)

\begin{tabular}{llll}
\hline Parameters & Balanced shoulder & Imbalanced shoulder & P \\
\hline Postfusion & $3.4 \pm 2.7$ & $5.8 \pm 3.3$ & $\mathbf{0 . 0 8 2}$ \\
Follow-up & $1.9 \pm 0.9$ & $4.3 \pm 2.6$ & $\mathbf{0 . 0 4 4}$ \\
T1T $\left(^{\circ}\right)$ & & & 0.900 \\
Preimplantation & $18.8 \pm 15.9$ & $17.9 \pm 15.1$ & 0.846 \\
Postimplantation & $12.7 \pm 10.5$ & $13.6 \pm 11.8$ & 0.822 \\
Prefusion & $16.1 \pm 13.1$ & $14.9 \pm 9.5$ & 0.523 \\
Postfusion & $15.0 \pm 15.1$ & $19.3 \pm 13.5$ & 0.295 \\
Follow-up & $11.3 \pm 11.8$ & $16.5 \pm 7.2$ & \\
\hline
\end{tabular}

$R S H$ radiographic shoulder height; $R S B$ radiographic shoulder balance; UIV upper instrumented vertebrae; LIV lower instrumented vertebrae; C7PL cervical 7 plumb line; CSVL centre sacral vertical line; SVA sagittal vertebral axis; MC major curve; PTC,proximal thoracic curve Bold numbers indicate $P<0.10$

and analyse the shoulder-balancing effect in a relatively large number of GR graduates who had completed final fusion, indicating that final fusion provided the opportunity to readjust the prefusion shoulder imbalance. Furthermore, the current results provide a wake-up call for spine surgeons to focus on the prefusion shoulder balance of GR graduates and to consider the importance of achieving shoulder balance in patients early after fusion. In practice, shoulder balance may not garner as much attention as spinal deformity correction or complication intervention. Extensive literature and a consensus on the surgical strategy for GR implantation and final fusion are still lacking. However, under the current conditions, the treatment results were satisfactory.

\section{Conclusions}

Shoulder balance could be improved by GR implantation but often changes during the multiple lengthening processes, and the final result is relatively unpredictable. Final fusion could further adjust the prefusion shoulder imbalance. Focusing on the prefusion shoulder balance of GR graduates and providing patients with early shoulder balance after fusion might be necessary.

\section{Abbreviations \\ GR: Growing-rod; growing rod(s); Imp: Implantation; RSH: Radiographic shoulder height; RSB: Radiographic shoulder balance; T1T: T1 tilt angle; CA: Clavicle angle; UIV: Upper instrumented vertebrae; LIV: Lower instrumented vertebrae; C7PL: Cervical 7 plumb line; CSVL: Centre sacral vertical line; SVA: Sagittal vertebral axis; PTC: Proximal thoracic curve; MC: Major curve; TK: Thoracic kyphosis; LL: Lumbar lordosis}

\section{Supplementary Information}

The online version contains supplementary material available at https://doi. org/10.1186/s12891-021-04221-9.

\section{Additional file 1}

\section{Acknowledgements}

We gratefully acknowledge Dr Qianqian Wang, PhD, Stanford University, California, USA, for her guidance on the research methods, Dr Yufu Liu, PhD,
Tsinghua University, Beijing, China, for his technical support, and Ms Qionghui He, MA, University College London.

\section{Authors' contributions}

The contributions of the authors are as follows: ZL: acquisition and analysis of data, drafting of the article, and statistical analysis; TL: analysis of data, drafting and revision of the article, and technical support; $\mathrm{YH}$ : design, review and revision of the final manuscript, technical and administrative support; LW: statistical analysis and drafting of the article; JJH: data acquisition and analysis; KG: acquisition and analysis of data; XG: drafting and revision of the article and technical support; HY: acquisition and analysis of data; NK: acquisition of data, revision of the article and review of the final manuscript; and FZ: revision of the article and review of the final manuscript. The author(s) read and approved the final manuscript.

\section{Funding}

No funds were received in support of this work.

\section{Availability of data and materials}

The datasets used and/or analysed during the current study are available from the corresponding author on reasonable request.

\section{Declarations}

\section{Ethics approval and consent to participate}

This study was approved by Beijing Chaoyang Hospital, Capital Medical University Ethics Committee Office. All authors confirm and declare that this study was conducted in compliance with the Declaration of Helsinki. Since some of the patients were under the age of 16, all patients or their parents agreed with the data and publication of the manuscript, and all participants or parents provided written informed consent.

\section{Consent for publication}

Not applicable.

\section{Competing interests}

The authors declare that they have no competing interests.

\section{Author details}

${ }^{1}$ Department of Orthopedic Surgery, Beijing Chaoyang Hospital, Capital Medical University, Gongti North Rd, No. 8, Beijing 100020, China. ${ }^{2}$ Karolinska Institutet, Stockholm, Sweden. ${ }^{3}$ The High School Affiliated to Renmin University of China, Beijing, China. ${ }^{4}$ Capital Medical University, Beijing, China. ${ }^{5}$ University of Wisconsin Madison, Madison, WI, USA.

Received: 13 November 2020 Accepted: 30 March 2021 Published online: 14 April 2021

\section{References}

1. Lenke LG, Dobbs MB. Management of juvenile idiopathic scoliosis. In: Journal of Bone and Joint Surgery - Series A; 2007. 
2. Zhang SF, Zhang L, Feng XM, Yang HL. Incidence and risk factors for postoperative shoulder imbalance in scoliosis: a systematic review and meta-analysis. Eur Spine J. 2018;27(2):358-69. https://doi.org/10.1007/ s00586-017-5289-y.

3. Qiu XS, Ma WW, Li WG, Wang B, Yu Y, Zhu ZZ, et al. Discrepancy between radiographic shoulder balance and cosmetic shoulder balance in adolescent idiopathic scoliosis patients with double thoracic curve. Eur Spine J. 2009; 18(1):45-51. https://doi.org/10.1007/s00586-008-0833-4.

4. Lee CS, Hwang CJ, Lim EJ, Lee DH, Cho JH. A retrospective study to reveal factors associated with postoperative shoulder imbalance in patients with adolescent idiopathic scoliosis with double thoracic curve. J Neurosurg Pediatr. 2016.

5. Trobisch PD, Samdani AF, Pahys JM, Cahill PJ. Postoperative trunk shift in Lenke 1 and 2 curves: how common is it? And analysis of risk factors. Eur Spine J. 2011;20(7):1137-40. https://doi.org/10.1007/s00586011-1820-8

6. Cao K, Watanabe K, Hosogane N, Toyama Y, Yonezawa I, Machida M, et al. Association of postoperative shoulder balance with adding-on in lenke type ii adolescent idiopathic scoliosis. Spine (Phila Pa 1976). 2014.

7. Arlet V, Marchesi D, Papin P, Aebi M. Decompensation following scoliosis surgery: Treatment by decreasing the correction of the main thoracic curve or "letting the spine go." Eur Spine J 2000.

8. Terheyden $\mathrm{JH}$, Wetterkamp M, Gosheger G, Bullmann V, Liljenqvist U, Lange $T$, et al. Predictors of shoulder level after spinal fusion in adolescent idiopathic scoliosis. Eur Spine J. 2018;27(2):370-80. https://doi.org/10.1007/ s00586-017-5210-8.

9. Yagi M, Takemitsu M, Machida M. Chest cage angle difference and rotation of main thoracic curve are independent risk factors of postoperative shoulder imbalance in surgically treated patients with adolescent idiopathic scoliosis. Spine (Phila Pa 1976). 2013.

10. Namikawa T, Matsumura A, Kato M, Hayashi K, Nakamura H. Radiological assessment of shoulder balance following posterior spinal fusion for thoracic adolescent idiopathic scoliosis. Scoliosis. 2015.

11. Elfiky TA, Samartzis D, Cheung W-Y, Wong Y-W, Luk KDK, Cheung KMC. The proximal thoracic curve in adolescent idiopathic scoliosis: surgical strategy and management outcomes. Glob Spine J. 2011;1(1):027-36. https://doi. org/10.1055/s-0031-1296054.

12. Hardesty CK, Huang RP, El-Hawary R, Samdani A, Hermida PB, Bas T, et al. Early-onset scoliosis: updated treatment techniques and results. Spine Deform. 2018;6(4):467-72. https://doi.org/10.1016/j.jspd.2017.12.012.

13. Skaggs DL, Akbarnia BA, Flynn JM, Myung KS, Sponseller PD, Vitale MG. A classification of growth friendly spine implants. J Pediatr Orthop. 2014

14. Bin ZY, Zhang JG. Treatment of early-onset scoliosis: Techniques, indications, and complications. Chin Med J (Engl). 2020;133:351-7.

15. Akbarnia BA, Breakwell LM, Marks DS, McCarthy RE, Thompson AG, Canale SK, et al. Dual growing rod technique followed for three to eleven years until final fusion: The effect of frequency of lengthening. Spine (Phila Pa 1976). 2008

16. Flynn JM, Tomlinson LA, Pawelek J, Thompson GH, McCarthy R, Akbarnia BA. Growing-rod graduates: lessons learned from ninety-nine patients who completed lengthening. J Bone Jt Surg - Ser A. 2013;95(19):1745-50. https:// doi.org/10.2106/JBJS.L.01386.

17. Üzümcügil $O$, Atici Y, Ozturkmen Y, Yalcinkaya M, Caniklioglu M. Evaluation of shoulder balance through growing rod intervention for early-onset scoliosis. J Spinal Disord Tech. 2012;25(7):391-400. https://doi.org/10.1097/ BSD.0b013e318227b52d.

18. Atici Y, Akman YE, Balioglu MB, Erdogan S. A comparison of the effects of two different techniques on shoulder balance in the treatment of congenital scoliosis: vertical expandable prosthetic titanium rib and dual growing rod. J Craniovertebr Junction Spine. 2015;6(4):190-4. https://doi. org/10.4103/0974-8237.167880.

19. Atici Y, Balioglu M, Akman Y, Albayrak A, Kargin D, Arikan Y, et al. Comparative analysis of shoulder balance through growth guidance techniques in the surgical treatment of early-onset scoliosis. J Spine. 2015; 04(01). https://doi.org/10.4172/2165-7939.1000201.

20. Akbarnia BA, Marks DS, Boachie-Adjei O, Thompson AG, Asher MA. Dual growing rod technique for the treatment of progressive early-onset scoliosis: a multicenter study. Spine. 2005;30:S46-57. https://doi.org/10.1097/ 01.brs.0000175190.08134.73.

21. Helenius IJ, Oksanen HM, McClung A, Pawelek JB, Yazici M, Sponseller PD, et al. Outcomes of growing rod surgery for severe compared with moderate early- onset scoliosis: a matched comparative study. Bone Jt J. 2018;100-B(6):772-9. https://doi.org/10.1302/0301-620X.100B6.BJJ-2017-1490.R1.

22. Helenius IJ, Sponseller PD, McClung A, Pawelek JB, Yazici M, Emans JB, et al. Surgical and Health-related Quality-of-Life Outcomes of Growing Rod "Graduates" With Severe versus Moderate Early-onset Scoliosis. Spine (Phila Pa 1976). 2019.

23. Kuklo TR, Lenke LG, Won DS, Graham EJ, Sweet FA, Betz RR, et al. Spontaneous proximal thoracic curve correction after isolated fusion of the main thoracic curve in adolescent idiopathic scoliosis. Spine (Phila Pa 1976). 2001.

24. Hong JY, Suh SW, Modi HN, Yang JH, Park SY. Analysis of factors that affect shoulder balance after correction surgery in scoliosis: a global analysis of all the curvature types. Eur Spine J. 2013;22(6):1273-85. https://doi.org/10.1007/ s00586-013-2697-5.

25. Sanders JO, Harrast JJ, Kuklo TR, Polly DW, Bridwell KH, Diab M, et al. The Spinal Appearance Questionnaire: Results of reliability, validity, and responsiveness testing in patients with idiopathic scoliosis. Spine (Phila Pa 1976). 2007.

26. Koo TK, Li MY. A guideline of selecting and reporting Intraclass correlation coefficients for reliability research. J Chiropr Med. 2016;15(2):155-63. https:// doi.org/10.1016/j.jcm.2016.02.012

27. Tang X, Luo X, Liu C, Fu J, Yao Z, Du J, et al. The spontaneous development of cosmetic shoulder balance and shorter segment fusion in adolescent idiopathic scoliosis with lenke i curve. Spine (Phila Pa 1976). 2016.

28. Clement RC, Anari J, Bartley CE, Bastrom TP, Shah R, Talwar D, et al. What are normal radiographic spine and shoulder balance parameters among adolescent patients? Spine Deform. 2020;8(4):621-7. https://doi.org/10.1007/ s43390-020-00074-9.

29. Chang DG, Kim JH, Kim SS, Lim DJ, Ha KY, Suk S II. How to improve shoulder balance in the surgical correction of double thoracic adolescent idiopathic scoliosis. Spine (Phila Pa 1976). 2014.

30. Yang JS, McElroy MJ, Akbarnia BA, Salari P, Oliveira D, Thompson GH, et al. Growing rods for spinal deformity: characterizing consensus and variation in current use. J Pediatr Orthop. 2010;30(3):264-70. https://doi.org/10.1097/ BPO.0b013e3181d40f94.

31. Thompson GH, Akbarnia BA, Kostial P, Poe-Kochert C, Armstrong DG, Roh J, et al. Comparison of single and dual growing rod techniques followed through definitive surgery: A preliminary study. Spine (Phila Pa 1976). 2005.

32. Dede O, Demirkiran G, Bekmez S, Sturm PF, Yazici M. Utilizing the stable-tobe vertebra saves motion segments in growing rods treatment for earlyonset scoliosis. J Pediatr Orthop. 2016;36(4):336-42. https://doi.org/10.1097/ BPO.0000000000000467

33. Yang X, Hu B, Song Y, Liu L, Zhou C, Zhou Z, et al. Coronal and sagittal balance in Lenke 5 AIS patients following posterior fusion: important role of the lowest instrument vertebrae selection. BMC Musculoskelet Disord. 2018; 19:1-10.

34. Rose PS, Lenke LG. Classification of operative adolescent idiopathic scoliosis: treatment guidelines. Orthop Clin North Am. 2007;38(4):521-9. https://doi. org/10.1016/j.ocl.2007.06.001.

35. Senkoylu A, Luk KDK, Wong YW, Cheung KMC. Prognosis of spontaneous thoracic curve correction after the selective anterior fusion of thoracolumbar/lumbar (Lenke 5C) curves in idiopathic scoliosis. Spine J. 2014;14(7):1117-24. https://doi.org/10.1016/j.spinee.2013.07.467.

36. Akel I, Pekmezci M, Hayran M, Genc Y, Kocak O, Derman O, et al. Evaluation of shoulder balance in the normal adolescent population and its correlation with radiological parameters. Eur Spine J. 2008;17(3):348-54. https://doi. org/10.1007/s00586-007-0546-0.

37. Vercauteren M, Van Beneden M, Verplaetse $R$, Croene P, Uyttendaele D, Verdonk R. Trunk asymmetries in a Belgian school population. Spine (Phila Pa 1976). 1982;7:555-62.

38. Gardner A, Berryman F, Pynsent P. What is the variability in shoulder, axillae and waist position in a group of adolescents? J Anat. 2017;231(2):221-8. https://doi.org/10.1111/joa.12630.

\section{Publisher's Note}

Springer Nature remains neutral with regard to jurisdictional claims in published maps and institutional affiliations. 